\title{
Changes in training posture induce changes in the chest wall movement and respiratory muscle activation during respiratory muscle training
}

\author{
Ju-Hyeon Jung ${ }^{1}$, Nan-Soo Kim ${ }^{2, *}$ \\ 'Department of Physical Therapy, Gimhae College, Gimhae, Korea \\ ${ }^{2}$ Department of Physical Therapy, College of Health Sciences, Catholic University of Pusan, Busan, Korea
}

Postural changes induce changes in chest wall kinematics and eventually pulmonary function, and affect chest wall shape and chest motion. This study aimed to examine the effects of postural change on changes in the chest wall during respiratory muscle training. Using a repeated measures design, this study followed 13 healthy adults (13 men; mean age, 23.73 years). All participants performed four postures (neutral, full trunk rotation, half-range trunk rotation, and lateral ribcage shift postures) during respiratory muscle training. The chest wall movement during the four postures was measured using a three-dimensional motion-analysis system during respiratory muscle training. Surface electromyography data were collected from the diaphragm and sternocleidomastoid muscles, and the asymmetric ratio of muscle activation was calculated based on the collected data. The chest wall movements of the upper costal and middle costal region were greater in the neutral posture than in the full rotation, half rotation, and lateral ribcage shift postures $(P<0.05)$. The respiratory muscle activation on diaphragm of left was greater in the full rotation posture than in the neutral posture, half rotation, and lateral ribcage shift postures $(P<0.05)$. The asymmetric ratio of muscle activation was greater in the full rotation posture than in the neutral posture, half rotation, and lateral ribcage shift postures $(P<0.05)$. This study verified that postural change during respiratory muscle training may affect chest wall movement and muscle activation. Thus, this study recommends respiratory muscle training to be performed in neutral posture.

Keywords: Respiratory muscle training, Chest wall movement, Respiratory muscle activation, Posture change

\section{INTRODUCTION}

Postural change leads to changes in spontaneous quiet breathing and affects thoraco-abdominal kinematics. The interaction among posture, patient sex, and rib cage and abdominal kinematics during quiet breathing in turn has an impact on chest wall kinematics (Romei et al., 2010; Sharp et al., 1975; Verschakelen and Demedts, 1995).

Two studies have reported that changes in body position alter pulmonary functions, and they have reported on the relative contributions of the rib cage and abdomen to ventilation (Romei et al., 2010; Sharp et al., 1975). Postural changes have been noted to impact chest wall shape, motion, and motion distribution be- tween compartments of the chest wall (Lee et al., 2010). Furthermore, thoracic rotation has been reported to decrease rib cage motion by altering rib articulations, the required intercostal muscle activity, and abdominal displacement (Lee et al., 2010; Lin et al., 2006).

Based on this theory, previous studies have confirmed the effects of postural change during quiet and deep breathing on chest wall volume and right and left chest volume (Aliverti et al., 2001), as well as a high correlation between postural changes and changes in vital capacity and quiet breathing (Verschakelen and Demedts, 1995). Previous studies have analyzed the effects of posture on chest wall kinematics and lung volume in various postures, including the supine and prone positions and a shift from the seated
${ }^{*}$ Corresponding author: Nan-Soo Kim (D) https://orcid.org/0000-0003-4694-8000 Department of Physical Therapy, College of Health Sciences, Catholic University of Pusan, 57 Oryundae-ro, Geumjeong-gu, Busan 46252, Korea Tel: +82-51-510-0575, Fax: +82-51-510-0578, E-mail: hnskim@cup.ac.kr Received: July 30, 2018 / Accepted: September 6, 2018
This is an Open Access article distributed under the terms of the Creative Commons Attribution Non-Commercial License (http://creativecommons.org/licenses/by-nc/4.0/) which permits unrestricted non-commercial use, distribution, and reproduction in any medium, provided the original work is properly cited. 
position to the supine position (Aliverti et al., 2001; Romei et al., 2010). In addition, the changes of chest wall volume in the lateral position have also been investigated (Nozoe et al., 2014). However, the changes in chest wall kinematics during the thoracic rotation and side-bending postures have not been compared. The thoracic rotation and side-bending postures are commonly utilized for partial expansion of the chest wall and chest mobilization in the clinical setting during training (Leelarungrayub et al., 2009).

Several portable respiratory muscle training devices have been developed in the last 20 years that are aimed to help patients with respiratory, cardiac, or neurological disorders to minimize or revert these alterations (Lima et al., 2014; McConnell and Romer, 2004). Among these devices, incentive spirometers and inspiratory muscle threshold trainers preserve airway patency by increasing respiratory muscle activity, such as the patients' diaphragm muscle and external intercostal muscles; they also act to increase the volume of the thoracic cavity, which forces air into the lungs (Xiao et al., 2012).

Recent reports have investigated the changes in chest wall volume in patients via chest wall kinematic analysis using respiratory muscle training devices and compared changes between the right and left chest wall volume. Through their analysis, the authors suggested that respiratory muscle training devices can enhance chest wall volume and reduce asymmetry (Lima et al., 2014). However, chest kinematic analyses that document the degree of expansion of specific areas of the chest wall through postural changes during respiratory muscle training have not been attempted.

Thus, this study aimed to examine the effects of postural change during respiratory muscle training on changes of the chest wall movement and respiratory muscle activation.

\section{MATERIALS AND METHODS}

\section{Participants}

Thirteen men with normal pulmonary function participated in the study. The subjects were screened according to the following inclusion and exclusion criteria. The inclusion criteria were: (a) absence of cardiac and pulmonary disease, (b) nonsmokers, (c) no endurance-trained athletes, and (d) age older than 18 years (Romei et al., 2010). The exclusion criteria were (a) subjects with neurological findings who had undergone surgery, (b) those currently receiving surgical treatment, and (c) those taking pain medications on a regular basis (Jung and Kim, 2016). The subjects' demographic characteristics are shown in Table 1.
Table 1. General characteristics of the subjects $(n=13)$

\begin{tabular}{lc}
\hline Characteristic & \multicolumn{1}{c}{ Mean \pm SD } \\
\hline Age $(\mathrm{yr})$ & $23.73 \pm 3.99$ \\
Height $(\mathrm{cm})$ & $175.00 \pm 4.91$ \\
Body weight $(\mathrm{kg})$ & $75.33 \pm 9.75$ \\
$\mathrm{FVC}(\mathrm{L})$ & $3.25 \pm 0.22$ \\
$\mathrm{FEV}_{1}(\mathrm{~L})$ & $2.93 \pm 0.24$ \\
$\mathrm{FEV}_{1} / \mathrm{FVC}$ & $89.86 \pm 4.62$ \\
$\mathrm{PEF}$ & $5.37 \pm 0.83$ \\
$\mathrm{MIP}\left(\mathrm{cmH}_{2} \mathrm{O}\right)$ & $95.94 \pm 20.13$
\end{tabular}

SD, standard deviation; FVC, forced vital capacity; $F V_{1}$, forced expiratory volume during the 1 second; PEF, peak expiratory flow; MIP, maximum inspiratory pressure.

All participants voluntarily provided consent to participate in this study. All protocols were approved by the Ethics Committee of the Catholic University of Pusan (CUPIRB-2015-008).

\section{Procedure}

Before testing, pulmonary function (forced vital capacity, forced expiratory volume in $1 \mathrm{sec}$, peak expiratory flow, and maximum inspiratory pressure [MIP]) was measured by using a spirometer (Pony Fx, Cosmed, Rome, Italy), and MIP was measured by using the Power Breathe K5 device (Power Breathe International Ltd., Warwickshire, UK).

Three-dimensional (3D) chest wall movement was measured and analyzed for four sitting positions: neutral, full trunk rotation to the left, half-range trunk rotation to the left, and lateral ribcage shift to the left posture (Kaneko and Horie, 2012; Lee et al., 2010) (Fig. 1).

Condition 1: neutral posture: refers to the "ideal" and "reference" postures; the subjects were asked to maintain thoracic kyphosis and lumbar lordosis and look straight ahead without head and neck tilt (A).

Condition 2: full trunk rotation posture; the subjects were asked to "turn as far as comfortable to the left and to maintain a full trunk rotation end range" (B).

Condition 3: half trunk rotation posture; after the subject performed full trunk rotation, half of the full rotation range was set and to maintain a half trunk rotation end range" $(\mathrm{C})$.

Condition 4: lateral shifting posture to the left posture; the subjects were asked to "let the left shoulder drop towards the hip and look straight ahead without head and neck tilt" (D).

Test orders were randomly assigned and each subject was asked to select a card. Then, applied to the subjects in order from the 

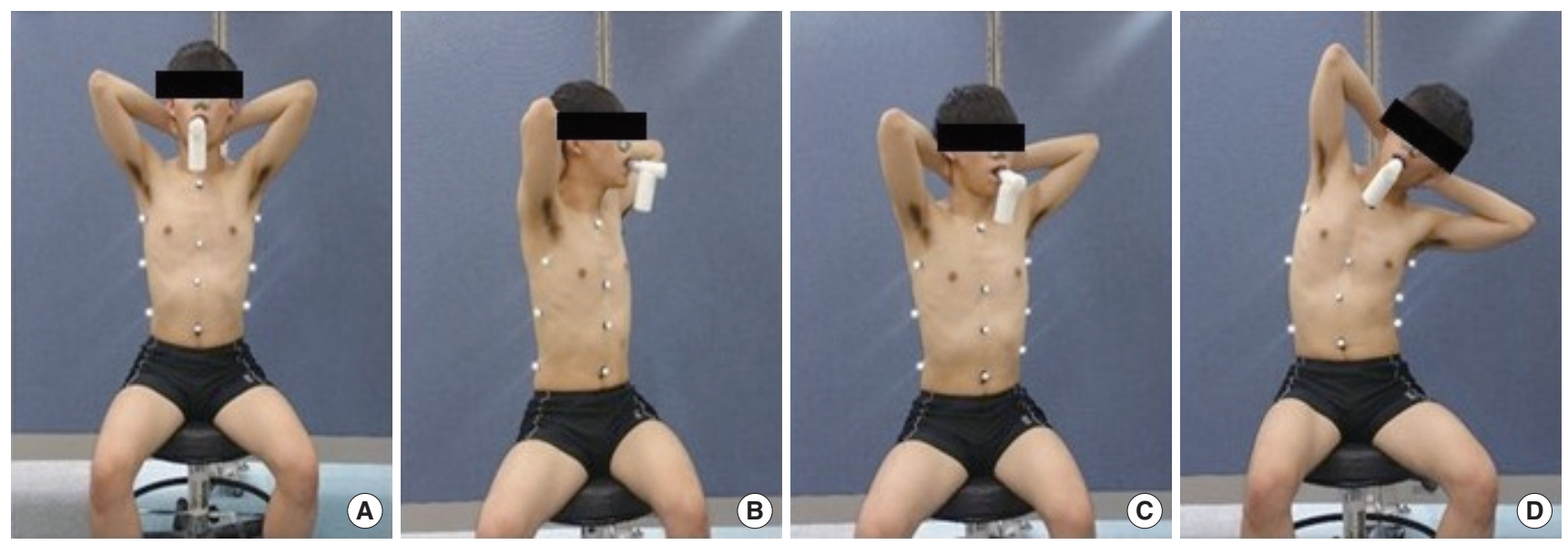

Fig. 1. Four training posture. (A) Neutral posture, (B) full trunk rotation to the left posture, (C) half-range trunk rotation to the left posture, and (D) lateral ribcage shift to the left posture.

card marked as one of the postures (Park et al., 2013). Once the testing began, the subjects wore a nose clip and respiratory muscle training device (Power Breathe medic classic; Power Breathe International Ltd., Warwickshire, UK) and breathed quietly. They performed the next stage of maximal inspiration through a mouthpiece until the respiratory valve was opened and air was let in, as instructed (Xiao et al., 2012). At each of the data collection points, maximal inspiratory efforts of 2- to 3-sec duration were performed 3 times in each posture, with an interval of at least 10 min between the postures. The chest wall movement and muscle activity outcomes were assessed concurrently (Hawkes et al., 2007; Paisani Dde et al., 2013).

\section{Inspiratory muscle loading}

A respiratory muscle training device was used to provide an acute bout of inspiratory muscle loading. The intensity was adjusted from the protocol used in a previous study (Hawkes et al., 2007), in which maximal inspiration at intensity of $30 \%$ of the MIP was measured prior to the experimental procedure (de Andrade et al., 2005).

\section{Chest wall movement}

A 3D motion-capture system (Oxford Metrics, Ltd., Oxford, UK; sampling rate $200 \mathrm{~Hz}$ ) was used to measure the amount of chest wall movement during respiratory muscle training. Fourteen sensors were placed over landmarks on the upper, middle, and lower ribcage and abdomen to measure chest wall movement (Lee et al., 2010) (Fig. 2).

The kinematic data were analyzed using Vicon Nexus software ver. 1.5.2 (Vicon Motion Systems Ltd., Oxford, UK). The mean value of the diameter of the sensors from three trials was used for the analysis (Lee et al., 2010). Chest wall movement was the change in these diameters from the end of inspiration of to the end of expiration (Lee et al., 2010). The end of inspiration and the end of expiration were determined in consideration of the distances between the sensors in each area with reference to the point at which the sum of the lateral diameter and anteroposterior diameter of the middle ribcage region reached maximum and minimum, respectively.

\section{Electromyographic analysis of the diaphragm and sternocleidomastoid}

The electromyographic (EMG) activities of the diaphragm and sternocleidomastoid on both sides were measured with a surface EMG system (Delsys Trigno Wireless EMG System; Delsys, Inc., Boston, MA, USA) with a sampling rate of 2,000 $\mathrm{Hz}$ and bandwidth of 20-450 Hz, obtained with chest wall movement analysis (Hawkes et al., 2007; Kang et al., 2015; Lima et al., 2014). All data were converted to root-mean-square (RMS) values.

To minimize skin impedance, the skin with shaving any hair and the skin swabbed with alcohol cotton before the electrodes were placed. Each EMG probe was attached to two superficial reusable bipolar electrodes consisting of $\mathrm{Ag} / \mathrm{AgCl}$ material and a conductive hydrogel adhesive. The electrodes were placed $2 \mathrm{~cm}$ apart from each other (Lima et al., 2014). The electrodes were placed on the diaphragm at the lowest intercostal spaces on both sides of the body and at the midclavicular line, and the sternocleidomastoid electrode was placed on the muscle body, $5 \mathrm{~cm}$ from the mastoid process (Hawkes et al., 2007; Lima et al., 2014) (Fig. 2). The diaphragm and sternocleidomastoid RMS values that were 


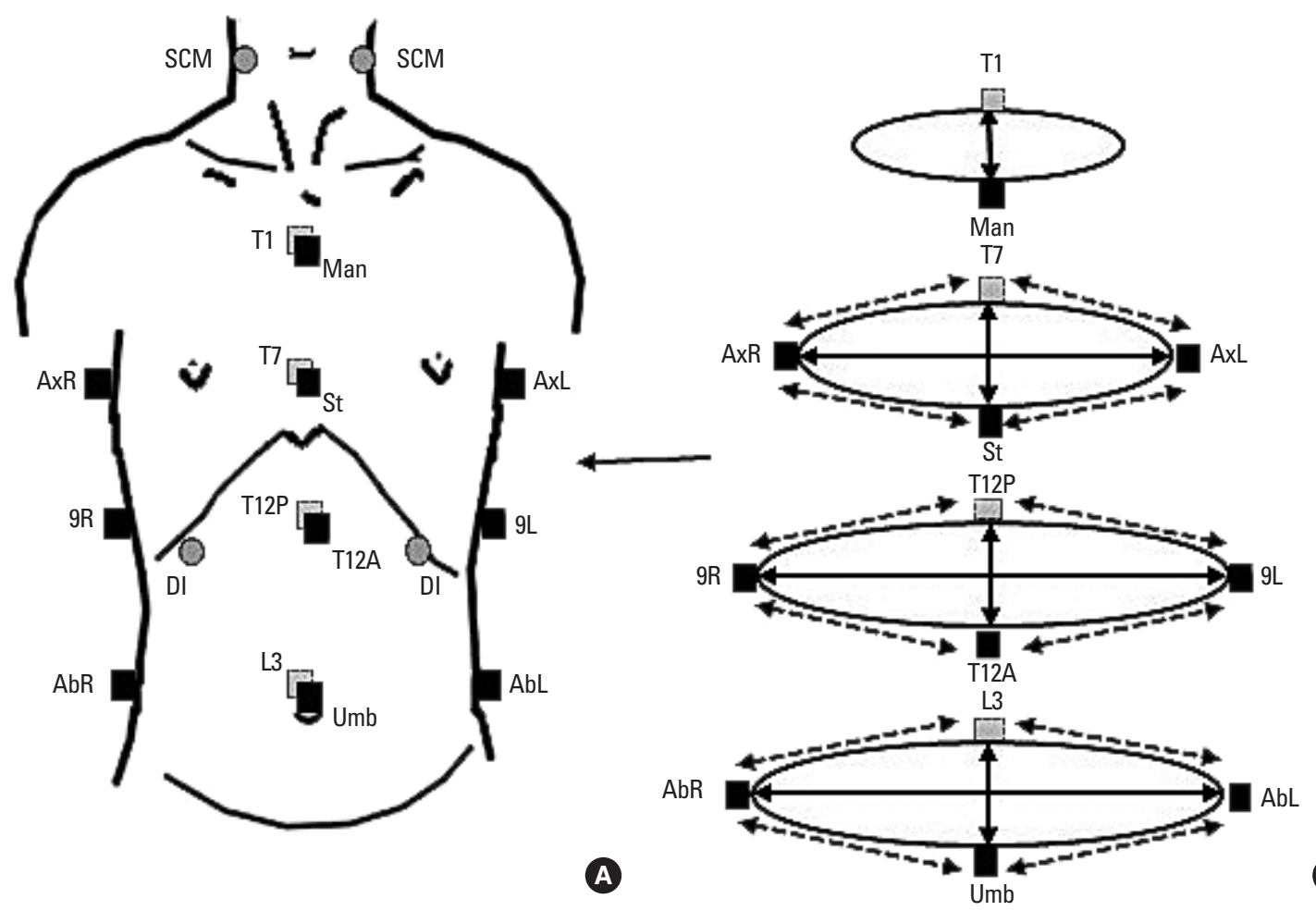

B

Fig. 2. (A) Schematic diagrams showing marker positioning on the chest wall and positions of electrode placement on the sternocleidomastoid (SCM), diaphragm (DI) of the subject. (B) Line drawings of diameters calculated from sensor position. Manubrium sensor (Man), sternum sensor (St), axilla sensors on the 4th rib (AxL, AxR), 9th rib sensors (9R, 9L), vertebrae sensor (T7, T12P, L3), anterior lower costal sensor on T12 level (T12A), abdominal sensor (AbR, AbL), umbilicus sensor (Umb).

Table 2. Magnitude of chest wall movement in the four postures

\begin{tabular}{lcccccc}
\hline Variable & Neutral & Full rotation & Half rotation & Lateral ribcage shift & Partial eta squared & $P$-value \\
\hline Chest wall $(\mathrm{mm})$ & & & & & & \\
UC & $5.57 \pm 3.19^{\mathrm{a})}$ & $3.36 \pm 2.95$ & $4.42 \pm 2.20^{\mathrm{a})}$ & $4.96 \pm 3.36^{\mathrm{a}}$ & 0.08 & $0.02^{*}$ \\
MC & $72.25 \pm 22.54^{\mathrm{a}, \mathrm{b}, \mathrm{c})}$ & $53.25 \pm 12.02$ & $62.06 \pm 16.83^{\mathrm{a})}$ & $59.93 \pm 16.87^{\mathrm{a}}$ & 0.26 & $0.02^{*}$ \\
LC & $81.44 \pm 30.02$ & $68.61 \pm 16.45$ & $74.71 \pm 26.34$ & $77.10 \pm 26.61$ & 0.08 & 0.06 \\
Abdominal $(\mathrm{mm})$ & $36.39 \pm 39.15^{\mathrm{c})}$ & $50.85 \pm 21.66$ & $31.52 \pm 42.86^{\mathrm{al}}$ & $52.07 \pm 27.12^{\mathrm{b}}$ & 0.06 & $0.01^{*}$ \\
\hline
\end{tabular}

Values are presented as mean \pm standard deviation.

UC, upper costal (diameter of man and T1); MC, middle costal (sum of diameters with AxL, AxR, St, and T7); LC, lower costal (sum of diameters with 9R, 9L, T12A, and T12P); Abdominal, sum of diameters with AbR, AbL, Umb, and L3.

${ }^{*} P<0.05$. alSignificantly different compared to the full rotation. ${ }^{b}$ Significantly different compared to the half rotation. ${ }^{\text {cl}}$ Significantly different compared to the lateral ribcage shift.

obtained via this method were entered into the following equation. A higher absolute value indicated a higher asymmetry ratio (Hsu et al., 2003). Asymmetry ratio=(1-[left side muscle RMS value/right side muscle RMS value]).

\section{Statistical analysis}

The sample size was calculated according to the data collected from 5 volunteers during respiratory muscle training. Considering a significance level of 0.05 and a statistical power of 0.80 , the op- timal number of subjects in the experiment was estimated as 10 . A distribution test was performed and the data showed normal distribution. One-way repeated-measures analysis of variance (ANOVA) of within factors and post hoc Fisher least-significant-difference (LSD) tests were conducted for each chest wall movement in the four conditions and one-way ANOVA and post hoc LSD tests were conducted for the amount of muscle activation (RMS) in the four conditions. The partial eta-squared values were included as an indicator of effect size when using ANOVA. The 
Table 3. Magnitude of respiratory muscle activation and asymmetry of muscle activation in the four postures

\begin{tabular}{|c|c|c|c|c|c|c|}
\hline Variable & Neutral & Full rotation & Half rotation & Lateral ribcage shift & $F$ & $P$-value \\
\hline \multicolumn{7}{|c|}{ Muscle activation (mV) } \\
\hline \multicolumn{7}{|l|}{$\mathrm{DI}$} \\
\hline Right & $36.66 \pm 17.93$ & $36.30 \pm 10.99$ & $35.30 \pm 15.67$ & $41.58 \pm 19.11$ & 0.38 & 0.76 \\
\hline Left & $42.82 \pm 13.67^{a)}$ & $74.33 \pm 50.57$ & $52.84 \pm 24.91$ & $40.89 \pm 12.98^{\mathrm{a})}$ & 3.45 & $0.02^{*}$ \\
\hline \multicolumn{7}{|l|}{ SCM } \\
\hline Right & $19.20 \pm 14.24$ & $11.97 \pm 7.13$ & $13.34 \pm 10.32$ & $31.56 \pm 37.27$ & 2.37 & 0.08 \\
\hline Left & $22.11 \pm 17.95$ & $42.42 \pm 67.62$ & $20.48 \pm 16.56$ & $20.46 \pm 14.13$ & 1.12 & 0.34 \\
\hline \multicolumn{7}{|c|}{ AR of muscle activation (ratio) } \\
\hline $\mathrm{DI}$ & $0.36 \pm 0.25^{a)}$ & $1.14 \pm 1.21$ & $0.78 \pm 0.75$ & $0.28 \pm 0.14^{\text {a) }}$ & 3.94 & $0.01^{*}$ \\
\hline SCM & $0.50 \pm 0.70$ & $2.03 \pm 2.82$ & $0.59 \pm 0.45$ & $0.73 \pm 0.91$ & 2.78 & 0.05 \\
\hline
\end{tabular}

Values are presented as mean \pm standard deviation.

DI, diaphragm; SCM, sternocleidomastoid; AR, asymmetric ratio.

${ }^{*} P<0.05$. al Significantly different compared to the full rotation.

data collected were analyzed using IBM SPSS Statistics ver. 21.0 (IBM Co., Armonk, NY, USA). Statistical significance was set at $P$-value of 0.05 .

\section{RESULTS}

Significant differences were found in chest wall movement in the upper costal region during the neutral, half rotation, lateral ribcage shift postures, and full rotation posture between conditions $(P<0.05)$ (Table 2$)$. Significantly greater chest wall movement was observed in the middle costal region in the neutral posture than in the full rotation, half rotation, and lateral ribcage shift postures $(P<0.05)$ (Table 2). Significant differences were found in chest wall movement in abdominal movement during the neutral, half rotation, lateral ribcage shift postures and full rotation posture between conditions $(P<0.05)$ (Table 2$)$.

Significant differences were found in muscle activation in the left diaphragm and asymmetry ratio of muscle activation in diaphragm during the neutral, half rotation, lateral ribcage shift postures, and full rotation posture between conditions $(P<0.05)$ (Table 3).

\section{DISCUSSION}

In this study, we hypothesized that postural change during respiratory muscle training will alter chest wall expansion, and we attempted to verify the optimal posture for increasing chest wall mobility during respiratory muscle training by analyzing four training postures that were suggested by a previous study (Lee et al., 2010). Existing studies on respiratory muscle training have mostly focused on improving pulmonary function through training intensity, training periods, and training (Pollock et al., 2013; Xiao et al., 2012). However, the authors of the previous studies predicted that the training posture would affect overall chest wall mobility and muscle activation, eventually leading to changes in pulmonary function, which supports the findings of the current study.

Minor changes in posture have been reported to alter muscle activation and joint orientation to preserve continuous respiratory function, and the altered chest wall mobility in specific areas manifests as limited overall chest wall mobility or problems with flexibility of the respiratory apparatus (Lee et al., 2010). Furthermore, changes in postural alignment have been found to alter range of movement and affects coupling patterns of thoracic spine and rib articulations, subsequently inducing changes in articular movement for respiration (Edmondston et al., 2007).

The present study found evidence supporting that of previous studies. In all areas on the chest wall (the upper, middle, and lower costal regions), the neutral posture was associated with the highest chest wall mobility, while the full rotation posture led to limited chest wall mobility. This might be due to the fact that the ribcage becomes impacted as the trunk rotates around the spine and articular motion decreases during respiration, reducing chest wall compliance (Lee et al., 2010; Lee, 2015). Moreover, in the result of the analysis of each segment of the chest wall, the full rotation posture showed more limitations in movement in the upper and middle costal regions than the other postures. Meanwhile, the upper and middle costal regions were limited, with relatively higher movement in the lower costal region than in the lateral ribcage shifting posture, which was similar to the movement in 
the neutral posture. The limited movement in the middle costal region during the full rotation posture may be explained by biomechanical data in which the midthorax (T3-6) primarily contributes to spinal rotation (Panjabi, 1992). Although the lateral ribcage shifting posture, in which the trunk is laterally bent at the eighth rib, limited the movement of the upper and middle regions of the chest wall, it showed similar movement as the neutral posture, as there was free chest wall movement in the lower costal region below the ninth rib. Although there were no significant differences in the lower costal region movements between the lateral ribcage shifting posture and full rotation posture, these results imply that postural change is involved in expanding specific regions of the chest wall.

Adjusted posture alters trunk muscle activity and affects ribcage and abdominal compliance (Callaghan and Dunk, 2002; Lee et al., 2010; O'sullivan et al., 2002). Previous studies have reported that trunk rotation stimulates activity in the intercostal and abdominal muscles of both sides of the body (Andersson et al., 2002; Rimmer et al., 1995), and that the ribcage and abdominal compliance are affected by altered intercostal and abdominal muscle contributions when trunk rotation postures, such as half and full rotation postures, are performed (Lee et al., 2010). The results of this study also revealed that the full rotation posture led to limited movement in the chest wall region but increased movement in the abdominal region, presumably due to reduced ribcage compliance, which altered diaphragm activity and increased contributions from the abdominal muscles (Cappello and De Troyer, 2004; Lee et al., 2010). Trunk rotation postures lower ribcage compliance and increase diaphragm activation (Cappello and De Troyer, 2004; Lee et al., 2010), but the overall lung volume is not expanded due to a lack of symmetrical muscle contraction. This study also verified that changes in diaphragm and sternocleidomastoid activity that were caused by rotation postures contributed to increasing the asymmetric ratio for diaphragm and sternocleidomastoid activation.

The results of this study should be interpreted in consideration of its limitations. The effects we investigated were short-term, thus, our study cannot confirm whether the effects will persist in the long term.

In summary, the findings of this study revealed that postural change during respiratory muscle training may affect chest wall movement. Particularly, because the rotation posture may limit chest wall movement and induce diaphragm asymmetry during training, we recommend clinical respiratory muscle training to be performed in neutral postures to improve postural alignment.

\section{CONFLICT OF INTEREST}

No potential conflict of interest relevant to this article was reported.

\section{REFERENCES}

Aliverti A, Dellacà R, Pelosi P, Chiumello D, Gatihnoni L, Pedoti A. Compartmental analysis of breathing in the supine and prone positions by optoelectronic plethysmography. Ann Biomed Eng 2001;29:60-70.

Andersson EA, Grundström H, Thorstensson A. Diverging intramuscular activity patterns in back and abdominal muscles during trunk rotation. Spine (Phila Pa 1976) 2002;27:E152-160.

Callaghan JP, Dunk NM. Examination of the flexion relaxation phenomenon in erector spinae muscles during short duration slumped sitting. Clin Biomech (Bristol, Avon) 2002;17:353-360.

Cappello M, De Troyer A. Role of rib cage elastance in the coupling between the abdominal muscles and the lung. J Appl Physiol (1985) 2004:97:85-90.

de Andrade AD, Silva TN, Vasconcelos H, Marcelino M, RodriguesMachado MG, Filho VC, Moraes NH, Marinho PE, Amorim CF. Inspiratory muscular activation during threshold therapy in elderly healthy and patients with COPD. J Electromyogr Kinesiol 2005;15:631639.

Edmondston SJ, Aggerholm M, Elfving S, Flores N, Ng C, Smith R, Netto $\mathrm{K}$. Influence of posture on the range of axial rotation and coupled lateral flexion of the thoracic spine. J Manipulative Physiol Ther 2007;30: 193-199.

Hawkes EZ, Nowicky AV, McConnell AK. Diaphragm and intercostal surface EMG and muscle performance after acute inspiratory muscle loading. Respir Physiol Neurobiol 2007;155:213-219.

Hsu AL, Tang PF, Jan MH. Analysis of impairments influencing gait velocity and asymmetry of hemiplegic patients after mild to moderate stroke. Arch Phys Med Rehabil 2003;84:1185-1193.

Jung JH, Kim NS. Relative activity of respiratory muscles during prescribed inspiratory muscle training in healthy people. J Phys Ther Sci 2016;28:1046-1049.

Kaneko H, Horie J. Breathing movements of the chest and abdominal wall in healthy subjects. Respir Care 2012;57:1442-1451.

Kang MH, Choi JY, Oh JS. Effects of crossed brassiere straps on pain, range of motion, and electromyographic activity of scapular upward rotators in women with scapular downward rotation syndrome. PM R 2015;7:1261-1268.

Lee DG. Biomechanics of the thorax - research evidence and clinical expertise. J Man Manip Ther 2015;23:128-138. 
Lee LJ, Chang AT, Coppieters MW, Hodges PW. Changes in sitting posture induce multiplanar changes in chest wall shape and motion with breathing. Respir Physiol Neurobiol 2010;170:236-245.

Leelarungrayub D, Pothongsunun P, Yankai A, Pratanaphon S. Acute clinical benefits of chest wall-stretching exercise on expired tidal volume, dyspnea and chest expansion in a patient with chronic obstructive pulmonary disease: a single case study. J Bodyw Mov Ther 2009; 13:338-343.

Lima IN, Fregonezi GA, Melo R, Cabral EE, Aliverti A, Campos TF, Ferreira GM. Acute effects of volume-oriented incentive spirometry on chest wall volumes in patients after a stroke. Respir Care 2014;59:11011107.

Lin F, Parthasarathy S, Taylor SJ, Pucci D, Hendrix RW, Makhsous M. Effect of different sitting postures on lung capacity, expiratory flow, and lumbar lordosis. Arch Phys Med Rehabil 2006;87:504-509.

McConnell AK, Romer LM. Respiratory muscle training in healthy humans: resolving the controversy. Int J Sports Med 2004;25:284-293.

Nozoe M, Mase K, Takashima S, Matsushita K, Kouyama Y, Hashizume H, Kawasaki Y, Uchiyama Y, Yamamoto N, Fukuda Y, Domen K. Measurements of chest wall volume variation during tidal breathing in the supine and lateral positions in healthy subjects. Respir Physiol Neurobiol 2014;193:38-42.

O'Sullivan PB, Grahamslaw KM, Kendell M, Lapenskie SC, Möller NE, Richards KV. The effect of different standing and sitting postures on trunk muscle activity in a pain-free population. Spine (Phila Pa 1976) 2002;27:1238-1244.

Paisani Dde M, Lunardi AC, da Silva CC, Porras DC, Tanaka C, Carvalho $\mathrm{CR}$. Volume rather than flow incentive spirometry is effective in im- proving chest wall expansion and abdominal displacement using optoelectronic plethysmography. Respir Care 2013;58:1360-1366.

Panjabi MM. The stabilizing system of the spine. Part I. Function, dysfunction, adaptation, and enhancement. J Spinal Disord 1992;5:383389.

Park KH, Ha SM, Kim SJ, Park KN, Kwon OY, Oh JS. Effects of the pelvic rotatory control method on abdominal muscle activity and the pelvic rotation during active straight leg raising. Man Ther 2013;18:220-224.

Pollock RD, Rafferty GF, Moxham J, Kalra L. Respiratory muscle strength and training in stroke and neurology: a systematic review. Int J Stroke 2013;8:124-130.

Rimmer KP, Ford GT, Whitelaw WA. Interaction between postural and respiratory control of human intercostal muscles. J Appl Physiol (1985) 1995;79:1556-1561.

Romei M, Mauro AL, D'Angelo MG, Turconi AC, Bresolin N, Pedotti A, Aliverti A. Effects of gender and posture on thoraco-abdominal kinematics during quiet breathing in healthy adults. Respir Physiol Neurobiol 2010;172:184-191.

Sharp JT, Goldberg NB, Druz WS, Danon J. Relative contributions of rib cage and abdomen to breathing in normal subjects. J Appl Physiol 1975;39:608-618.

Verschakelen JA, Demedts MG. Normal thoracoabdominal motions. Influence of sex, age, posture, and breath size. Am J Respir Crit Care Med 1995;151(2 Pt 1):399-405.

Xiao Y, Luo M, Wang J, Luo H. Inspiratory muscle training for the recovery of function after stroke. Cochrane Database Syst Rev 2012;(5): CD009360. 